\title{
BUFFER BASED ROUTING MECHANISM FOR LOAD BALANCING IN WIRELESS MESH NETWORKS
}

\author{
Keerthi D S \\ Department of Electronics and Communication Engineering, \\ Malnad College of Engineering, Hassan, India \\ Shobha Rani A \\ Department of Electronics and Communication Engineering, \\ BMS Institute of Technology and Management, Bengaluru, India

\section{T G Basavaraju} \\ Department of Computer Science and Engineering, \\ Govt. Sri Krishnarajendra Silver Jubilee Technological Institute, Bengaluru, India
}

\begin{abstract}
In recent years it is witnessed that the Wireless Mesh Networks (WMNs) are becoming the most promising technology as they offer low cost broadband wireless connectivity, larger coverage area, high flexibility and easy deployment. WMNs are an extension of existing wireless networks. WMN is an emerging technology; however, there are certain challenges that still exist in the network such as scalability, load balancing, mobility, power management etc. Here we have proposed a novel routing protocol which considers buffer occupancy of intermediate nodes for route selection. Simulation results convey that the proposed protocol outstandingly enhances the performance of the network by balancing the traffic load among less congested nodes compared to the standard protocol.
\end{abstract}

Keywords: Buffer, Load balancing, WMN.

Cite this Article: Keerthi D S, Shobha Rani A and T G Basavaraju, Buffer Based Routing Mechanism for Load Balancing in Wireless Mesh Networks. International Journal of Computer Engineering and Technology (IJCET), 10(1), 2019, pp. 1-10. $\mathrm{http}: / /$ iaeme.com/Home/issue/IJCET?Volume=10\&Issue $=1$ 


\section{INTRODUCTION}

WMNs are one of the most favorable wireless technologies which find several applications, e.g., broadband home networking, enterprise networking, community and neighborhood networks, building automation etc. They are an extension of wireless ad-hoc networks and they aim at delivering a cost effective and robust, high bandwidth network over a particular coverage area. WMNs [1] are dynamically self-healing, self-organized and self-configured as the nodes in the network automatically establish and maintain mesh connectivity among themselves which in turn provide benefits such as effortless network maintenance, low up-front costs and dynamic and reliable service coverage. A more sophisticated routing protocol along with an efficient routing metric can be used to enhance the performance of the WMNs. WMNs employ a number of routing protocols where every protocol maintains its own routing strategy $[2,3]$. These protocols are categorized as proactive, reactive and hybrid based on the strategies adopted by them.

Load Balancing is a method of evenly distributing the traffic across two or more nodes for effectively mediating communication and achieving redundancy even if there is any link failure. Load balancing can be achieved by increased throughput, minimal overheads and optimal utilization of resources. Whenever the routes are exploited repeatedly over a longer duration, the packet concentration elevates in intermediate nodes. This creates bottlenecks and thereby degrades the network performance due to congestion and also results in longer delays. The caching technique used in most of the on-demand routing protocols causes additional mass of load on certain nodes. In WMN, congestion is the major cause for the uneven distribution of traffic that causes underperformance of the network. Congestion is a condition where multiple packets are present in network exceeding existing resource available and thereby deteriorating the performance of the network. These packets completely fill up the buffer space and possibly overflow, manifesting in excess end to end delay, packet drop and low network throughput and degraded bandwidth.

The organization of the paper is as follows. The related work is discussed in Section 2. The proposed routing scheme BBL-AODV is completely described in section 3. The simulation results, performance comparison and analysis are presented in section 4 . The inference of the paper is in section 5 .

\section{RELATED WORK}

Many protocols exist for load balancing in wireless networks. Most of them have considered parameters like link occupancy, queue size, delay etc. as a metric for route selection. In [4], the authors have proposed a buffer aware routing protocol which takes buffer space of neighbouring nodes into account while taking routing decision. Even though the protocol performs well it introduces more delay while performing route discovery.

In [5], the authors present a congestion control approach by advertising the buffer space to the surrounding neighbour nodes. This approach is independent of the routing protocol but it needs additional mechanisms when inappropriate traffic is generated in the network. Congestion avoidance mechanism [6] deals with high load and fast mobility, which selects the best path based on minimum value of buffer queue occupancy instead of minimum hop count as in standard AODV.

Kruti N Kapadia et al. [7] have put forth an airtime congestion aware metric for a multiradio WMN. It uses airtime link metric for estimating RTT for delay calculation and channel utilization. With the combination of both parameters, load is uniformly distributed over less congested nodes. Raffaele Bruno et al. [8] have developed an algorithm based on queuing model for detection of congested paths in heterogeneous mesh networks. 
The algorithm is implemented at the nodes with the objective of forwarding traffic to less congested gateways based on their queue occupancy.

Dhurandher et al [9] have presented a novel protocol for distributed admission control and interference aware admission control for WMN with the aim of providing QoS guarantee for multimedia applications. The protocol calculates suitable admission control ratio that ensures loss rate within the tolerable limit. In addition to this it also computes end to end delay to exercise flow request. ECARP [10] is proposed for heavy traffic loads in MANETs and this protocol considers the number of packets in node buffer to regulate congestion among nodes. CARM [11] prefers less congested, high throughput links for traffic by employing weighted delay, retransmission count, and queuing delay in buffer.

In our work we have proposed a modified AODV protocol called Buffer Based Load Balancing Adhoc on Demand Protocol (BBL-AODV), which considers node buffer occupancy as well as average buffer occupancy of all neighbouring nodes as a routing metric during route discovery.

\section{BUFFER BASED LOAD BALANCING AODV PROTOCOL}

Buffer Based Load Balancing AODV Protocol (BBL-AODV) is an enhancement over the standard AODV protocol. It uses a path metric which allows source node to select the best path to the destination based on buffer occupancy of intermediate nodes. The standard AODV protocol discovers the route by broadcasting/flooding RREQ message and establishes a path when RREP message is received. The path selection is based on minimum hop count and sequence number irrespective of packet handling capacity. In Buffer Based Load Balancing (BBL) Protocol, each node maintains information about its node buffer occupancy in its routing table. Each node calculates its average buffer occupancy avg_buff_occ based on node buffer occupancy node buff occ of all neighboring nodes and itself. The node is assigned with a threshold value thr for optimum buffer utilization. This threshold value defines buffer space that a node requires to forward received packets. Whenever a node receives RREQ packet, it compares its current node buffer occupancy with threshold thr. If the current buffer occupancy is less than threshold (node_buff_occ $<$ thr), the node broadcasts RREQ packets or else it discards. Therefore, load is distributed along the less congested path and this enhances the performance of the wireless mesh network.

The average buffer occupancy is calculated as follows:

$$
\text { avg_buff_occ }=\frac{\text { node_buff_occ }+\sum_{j=1}^{n} \text { node_buff_occ }}{n+1}
$$

where ' $\mathrm{n}$ ' represents total neighboring nodes.

The node_buff_occ represents the buffer occupancy of the node which is given by the following equation.

$$
\text { node_buff_occ }=\frac{\sum_{i=1}^{b} b u f f_{i_{-}} p k t}{b}
$$

where, buff ${ }_{i-}$ pkt represent total number of packets in every buffer present.

' $b$ ' refers to the number of buffers in each node.

The BBL-AODV Algorithm for Route Selection is described as follows.

Step 1: Node has data to send

Step 2: Check the active nodes for transmission // active refers to a node already participating in data transfer 
Step 3: If ( node $==$ active)

then go to step 4

else go to step 7

end if

Step 4: Calculate node_buff_occ of each intermediate node.

Step 5: If node_buff_occ $<$ thr,

then forward the packet

else rebroadcast RREQ

end if

Step 6: Broadcast RREQ and wait for RREP

Step 7: Receive RREP go to step 4

\section{PERFORMANCE EVALUATION}

This section discusses parameters and performance metrics chosen for simulation of the proposed BBL-AODV protocol with standard AODV protocol. The performance of the proposed routing mechanism is evaluated by carrying out simulations using the licensed network simulator Qualnet v6.1.

\subsection{Simulation Parameters}

We have done substantial number of simulations for studying the performance and effectiveness of the suggested BBL-AODV against the standard AODV protocol in a wireless mesh network. The simulation is run for 300 seconds on a grid topology of simulation area $1500 \mathrm{x} 1500 \mathrm{~m}^{2}$ at lower and higher data rates for different Node densities. The nodes are placed in the simulation area in a uniform fashion with $150 \mathrm{~m}$ as inter-node distance. The traffic source selected is Constant Bit Rate traffic with each packet size being set to 512 bytes. The Table 1 below describes the simulation parameters employed for performance analysis.

Table 1 Simulation Parameters

\begin{tabular}{|c|c|}
\hline Parameter & Value \\
\hline Protocols & AODV, BBL-AODV \\
\hline No. of Nodes & $25,36,49,64,81,121$ \\
\hline Radio type & $802.11 \mathrm{~b}$ Radio \\
\hline MAC Protocol & $802.11 \mathrm{~s}$ \\
\hline Antenna Model & Omni-directional \\
\hline Path Loss Model & Two ray propagation \\
\hline Traffic Type & CBR \\
\hline No. of CBR & 3 \\
\hline
\end{tabular}

\subsection{Performance Metrics}

The effectiveness of the proposed and the standard protocol is compared using the performance metrics like throughput, total number of packets received, end to end delay and jitter.

- Throughput: The rate at which message is delivered successfully over a communication channel and is measured in bits per second.

- Total number of packets received: It is the number of packets received at the destination.

- End-to-end delay: It is the time taken by a packet to traverse from source to destination.

- Jitter: It is measuring time difference in packet inter-arrival time. 


\subsection{Simulation Results}

In this paper, the results are presented for different node densities at low and high data rates for both BBL-AODV and standard AODV protocol.

\subsubsection{Low Data rate}

In this scenario packets are forwarded through three CBR connections. Each source generates a low traffic of 1 packet/sec and is routed to the destination for a period of $300 \mathrm{sec}$. The above procedure is repeated for 36, 49, 64, 81 and 121 node densities respectively. After each simulation, the performance metrics such as throughput, delay, jitter and total messages received are recorded.

Figure.1-4 shows throughput, total packets received, end-to-end delay and jitter with varying node densities for BBL-AODV and AODV. It is observed that throughput remains almost same for BBL-AODV and standard AODV protocol. This is because the traffic generated in the network is within the buffer threshold limit for each node. So, both protocols use default routing strategy for path selection in the network. This is true even for total packets received, end-to-end delay and jitter respectively.

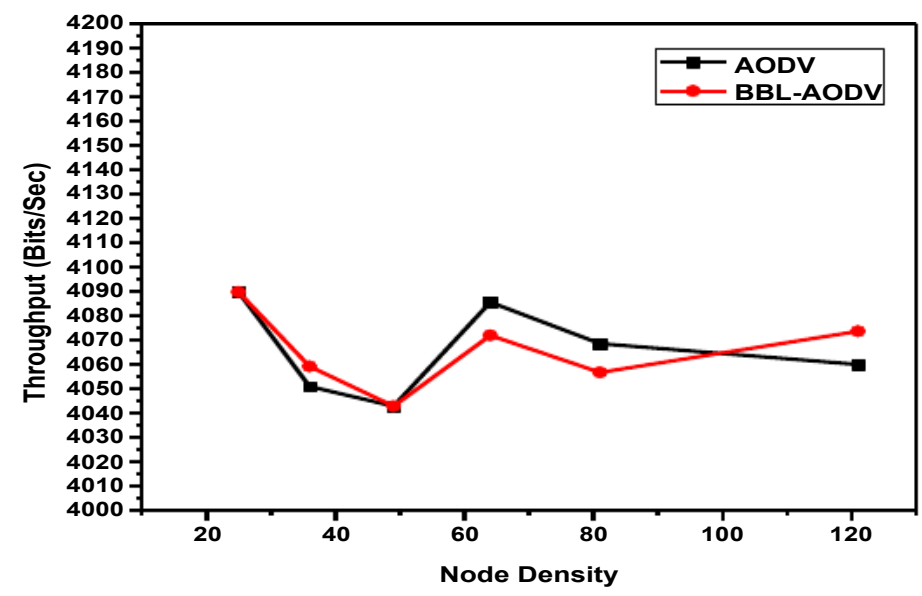

Figure 1 Variation of throughput v/s node densities

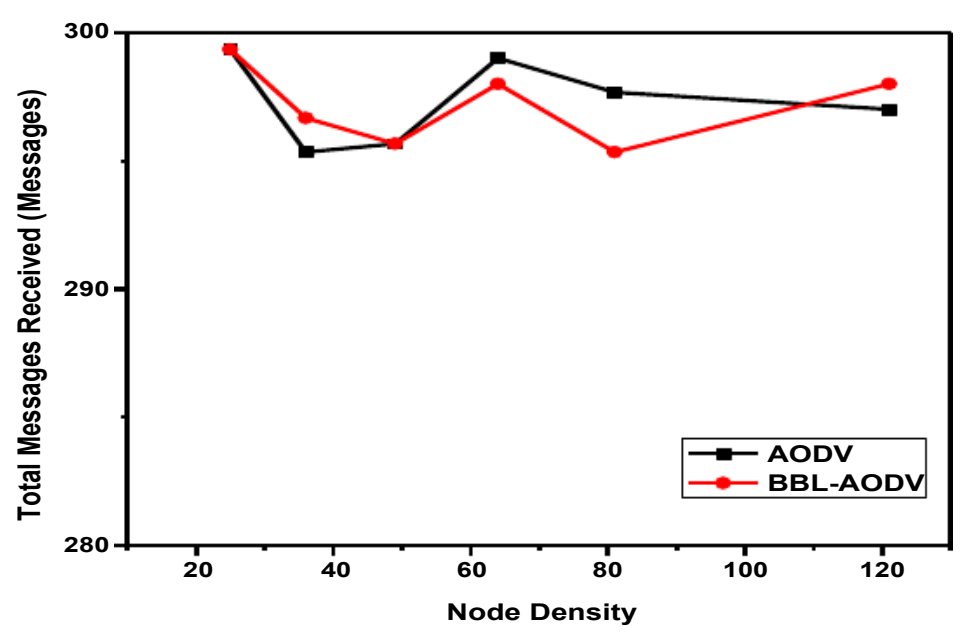

Figure 2 Variation of total messages received v/s node densities 


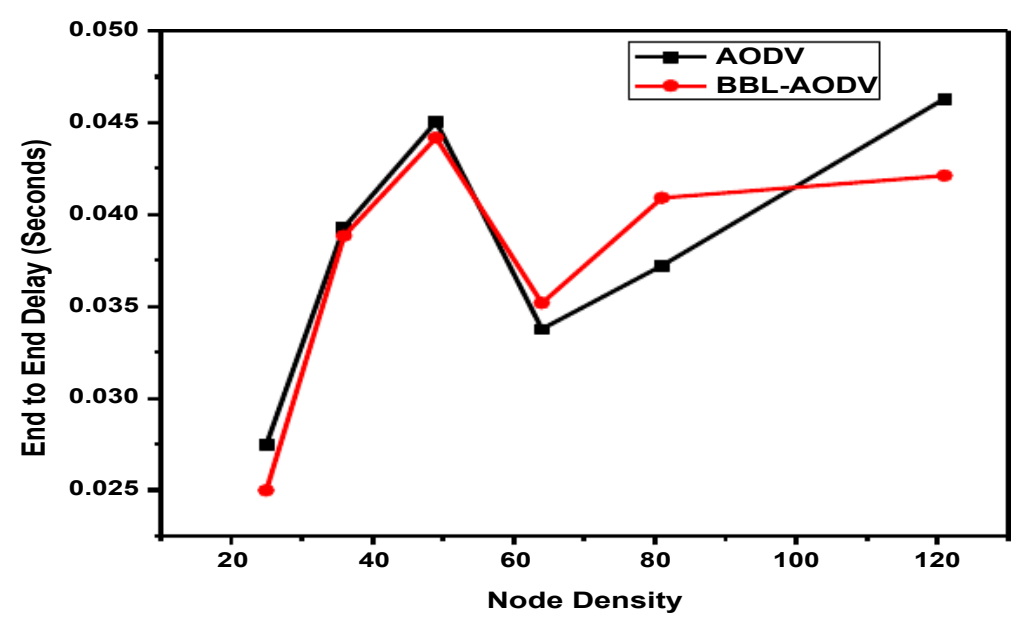

Figure 3 Variation of End to End Delay v/s node densities

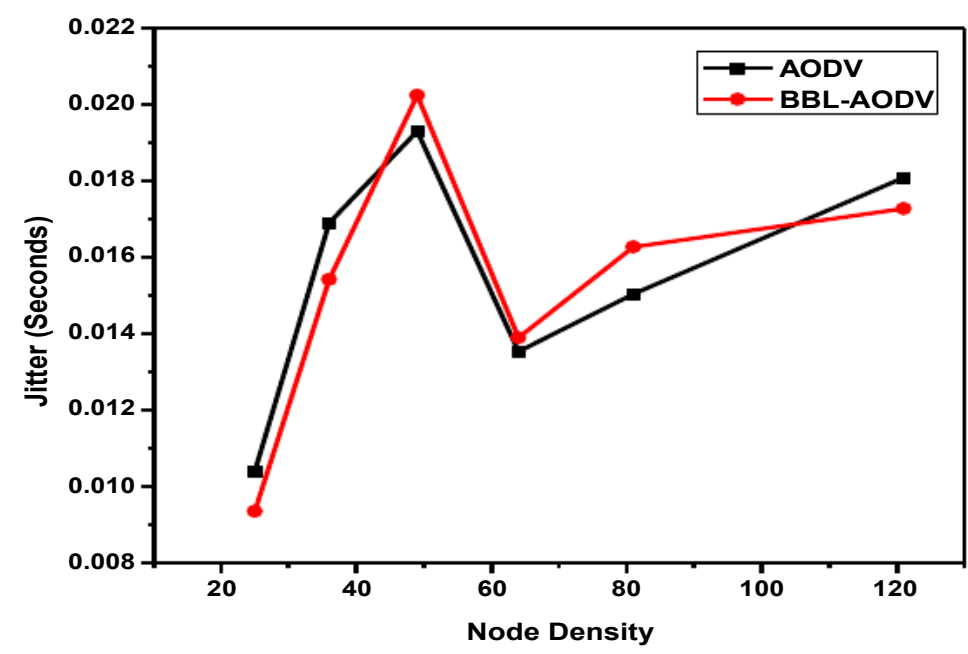

Figure 4 Variation of Jitter v/s node densities

\subsubsection{High Data rate}

In this section, the proposed protocol is compared with AODV protocol for a high data rate. The packets are forwarded through three CBR connections. Each source generates high traffic that is $100 \mathrm{packet} / \mathrm{sec}$ and is routed to the destination for a period of $300 \mathrm{sec}$. The above procedure is repeated for $36,49,64,81$ and 121 node densities respectively. After each simulation the performance metrics such as throughput, delay, jitter and total messages received are recorded.

Figure 5 shows the variation of throughput with respect to the node densities for BBLAODV and AODV protocol. It is observed that throughput for our proposed protocol is greater than AODV. This is due to the fact that when the load is very high, AODV failed to handle congestion thus resulting in degradation of the performance of the network. But the performance of the BBL-AODV is good since it uses the knowledge of buffer availability of each node during route discovery and avoids packet forwarding through the highly congested nodes. 
Figure 6 depicts the total message received v/s node density. It is evident that the number of packets received at the destination is more in BBL-AODV as compared to AODV. This is due to the fact that the packet drop ratio is less in BBL-AODV as it avoids nodes having buffer occupancy greater than threshold which in turn increases the packet reception at the destination.

In Figure 7 it can be observed that the end-to-end delay in case of BBL-AODV is less than that of AODV. As traffic flow increases, AODV will experience higher delay due to congestion. Therefore, data packets take more time to reach the destination. BBL-AODV captures the link quality by monitoring buffer utilization. It minimizes congestion along the path by computing buffer occupancy based on the proposed metric avg_buff_occ and node_buff_occ thus nodes are not over flooded. This results in optimum utilization of all network resources thereby allowing data packets to take less time to reach destination. Hence end to end delay of BBLAODV is lower than that of AODV. Since delay variation in BBL-AODV is less than AODV, it is suitable for real time applications. Hence BBL-AODV performs better than AODV protocol.

Figure 8 shows the jitter variation for BBL-AODV and AODV. It is observed that BBL_AODV experiences lesser jitter for increase in the node density and traffic rate when compared to AODV. Hence the proposed protocol outperforms the AODV protocol in terms of throughput, total number of packets received, end to end delay and jitter.

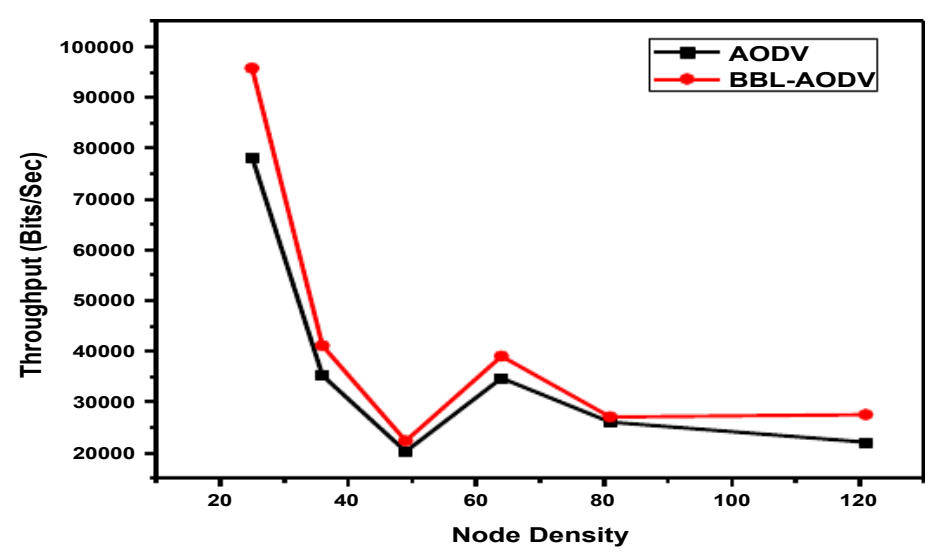

Figure 5 Variation of throughput $\mathrm{v} / \mathrm{s}$ node densities

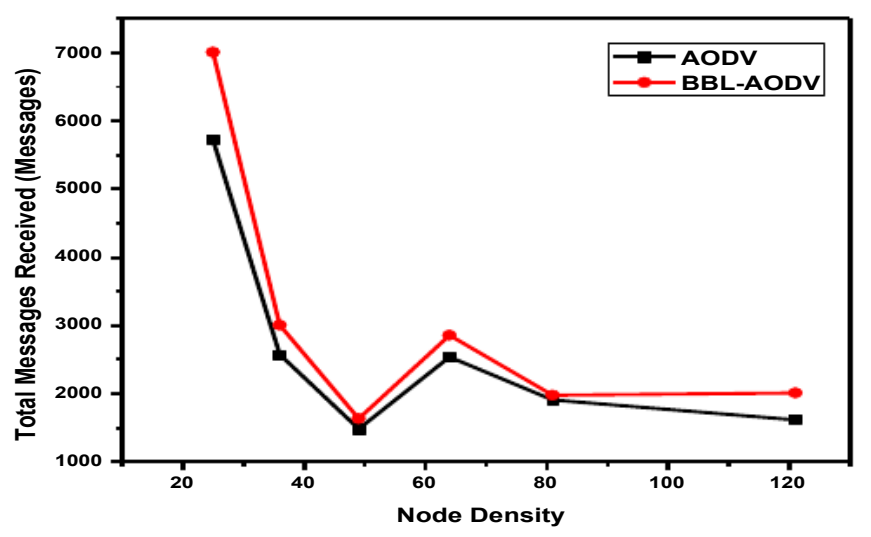

Figure.6. Variation of total messages received v/s node densities 


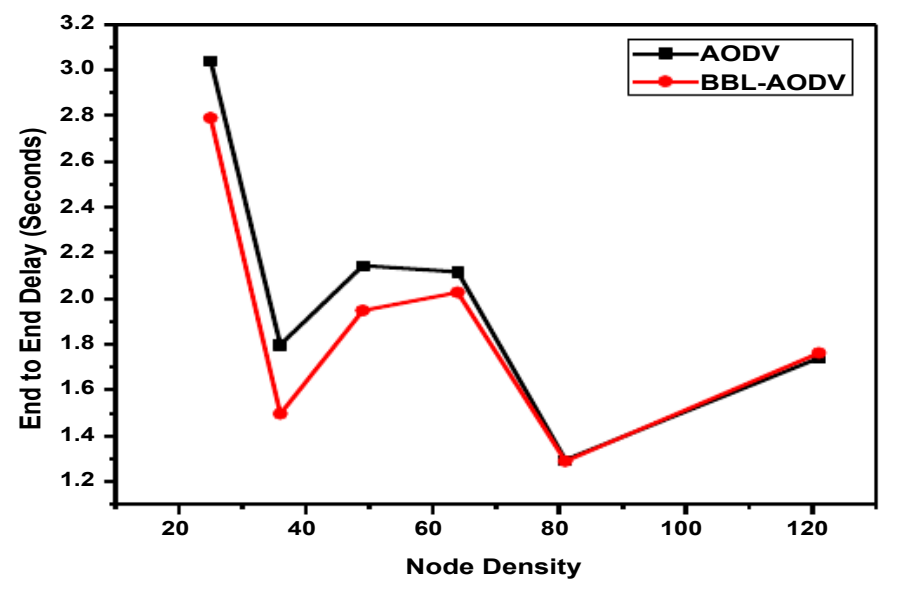

Figure 7 Variation of End to End Delay v/s node densities

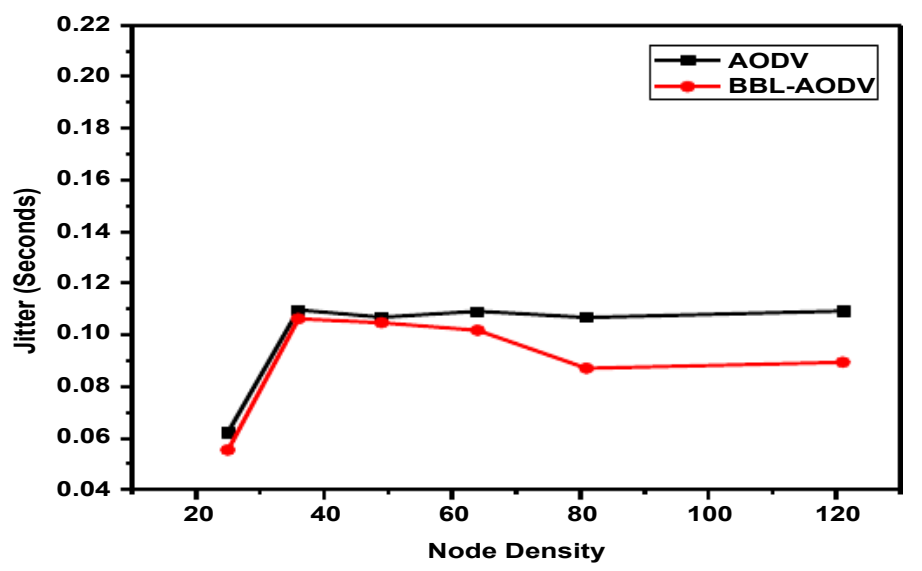

Figure 8 Variation of Jitter v/s node densities

\section{CONCLUSION}

In this work, we have proposed BBL-AODV; an effective buffer-based routing protocol for wireless mesh network. It captures link quality by monitoring buffer utilization. It minimizes congestion along the path by computing buffer occupancy based on the proposed routing metric. Simulations are done to analyze the proposed protocol's performance for different node densities with varying data rate. The metrics used to estimate the performance of the proposed protocol and standard protocol are throughput, delay, jitter and total messages received. From the results, it is evident that the proposed protocol outperforms the standard AODV protocol scheme with reference to the predefined performance metrics and hence results in optimum utilization of all network resources and increases the overall performance of the wireless mesh network.

\section{ACKNOWLEDGEMENT}

This work is supported by the Technical Education Quality Improvement Program Phase II with the World Bank assistance. The authors would like to thank State Project Facilitation Unit, Directorate of Technical Education, Government of Karnataka, National Project Implementation Unit, MHRD, New Delhi for their support and encouragement. 
Buffer Based Routing Mechanism for Load Balancing in Wireless Mesh Networks

\section{REFERENCES}

[1] F. Akyildiz, X. Wang, and W. Wang, Wireless mesh networks: a survey, Computer Networks Journal, Volume 47, 2005, pp.445-487.

[2] Eslami, Mohammad et al, A survey on wireless mesh networks: Architecture, specifications and challenges, 2014 IEEE $5^{\text {th }}$ Control and System Graduate Research Colloquium, 2014, pp.219-222.

[3] M. L. Sichitiu, Wireless mesh networks: Opportunities and challenge, Proc. of the Wireless World Congress, (Palo Alto, Ca), May 2005.

[4] Kamal Mistry, Sanjay Srivastava, R. B. Lenin, Buffer aware routing in interplanetary Ad Hoc Network, First International Communication Systems and Networks and Workshops, 2009, pp. 1- 10.

[5] Jani Lakkakorpi, Mikko Pitkänen and Jörg Ott, using buffer space advertisements to avoid congestion in mobile opportunistic DTNs" Proceedings of the 9th IFIP TC 6 International conference on Wired/wireless internet communications, 2011, pp. 386-397.

[6] Maroufi B.E., Rahmani M.D., Rziza M, Congestion Avoidance in AODV Routing Protocol Using Buffer Queue Occupancy and Hop Count, Proceedings of the Mediterranean Conference on Information \& Communication Technologies 2015, pp.193-200.

[7] Kruti N. Kapadia. Dayanand D. Ambawade, Congestion aware load balancing for multiradio Wireless Mesh Network, International Conference on Communication, Information \& Computing Technology (ICCICT) 2015.

[8] Raffaele Bruno, Marco Conti, Antonio Pinizzotto, A Queuing Modeling Approach for Load-Aware Route Selection in Heterogeneous Mesh Networks, IEEE International Symposium on a World of Wireless, Mobile and Multimedia Networks \& Workshops 2009.

[9] Sanjay Kumar Dhurandher, Isaac Woungang, Mohammad S. Obaidat, A Distributed Adaptive Admission Control Scheme for Multimedia Wireless Mesh Networks, IEEE Systems Journal Volume 9, Issue 2, June 2015, pp. 595 - 604.

[10] T. G. Basavaraju, S. K. Sarkar, C. Puttamadappa, and M. A. Gautham, ECARP: an efficient congestion adaptive routing protocol for mobile Ad hoc networks, in Proceedings of the 6th International Conference on ITS Telecommunications Proceedings, pp. 715-718, Chengdu, China, June 2006.

[11] X. Chen, H.M. Jones, and A. D. S. Jayalath, Congestion-aware routing protocol for mobile ad hoc networks, in Proceedings of IEEE Conference of Vehicular Technology, pp. 21-25, October 2007.

[12] Keerthi D S, T.G. Basavaraju, Load Balancing Routing Mechanisms for Wireless Mesh Networks: A Survey, in Fourth International Conference on Emerging Research in Computing, Information, Communication and Applications (ERCICA), 2016, Springer Proceedings.

[13] Sooyeol Yang, Youngmi Baek, Junhyung Kim, Keuchul Cho and Kijun Han, A routing metric for load balance in wireless mesh networks, $11^{\text {th }}$ International Conference on Advanced Communication Technology (ICACT) Volume 03, 2009, pp.1560-1565.

[14] Ekram Hossain, Kin K. Leung, Wireless Mesh Networks: Architectures and Protocols, Springer, 2008.

[15] Peipei Zhao, Jiansheng Qian, The Study of Wireless Mesh Network Routing Algorithm Based on Interference Model, Fourth International Conference on Genetic and Evolutionary Computing (ICGEC), 2010, pp.98-101.

[16] Benyamina D, Hafid A. and Gendreau M, Wireless Mesh Networks Design-A Survey, Communications Surveys \& Tutorials, IEEE, Volume 14, Issue 2, 2012, pp. 299 - 310.

[17] Guerin J, Portmann M and Pirzada A, Routing metrics for multi-radio wireless mesh networks, Telecommunication Networks and Applications Conference (ATNAC), Australasian, 2000, pp.343-348. 
[18] Bari S.M.S, Anwar F and Masud M.H, Performance study of hybrid Wireless Mesh Protocol (HWMP) for IEEE 802.11s WLAN mesh networks, International Conference on Computer and Communication Engineering (ICCCE), 2012, pp.712-716.

\section{BIOGRAPHY}

Keerthi D S. received his B.E. and M.Tech degrees from Visvesvaraya Technological University (VTU), Belagavi. He is pursuing Ph.D. under the guidance of Dr. T. G. Basavaraju at the Department of Computer Science and Engineering Research Centre, GSKSJ Technological Institute affiliated to VTU, Belagavi. He is currently working as an Assistant Professor at Malnad College of Engineering, Hassan, Karnataka. His research interest is in the field of Wireless Mesh Networks.

Shobha Rani A. received her B. E Electronics and Communication Engineering) degree from Bangalore University in the year 2000, M. Tech (Digital Electronics and Communication) Degree from Visvesvaraya Technological University (VTU), Belgaum in the year 2005, and $\mathrm{Ph}$. D (Networking) degree from Kuvempu University, Shimoga in the year, 2014. She is currently working as a Associate Professor in the Department of ECE at BMS Institute of Technology and Management, Bengaluru, India. She has 13 technical articles in good reputed journals and conferences. Her research interests include designing and implementing efficient protocols for wireless networks such as Ad-hoc, Sensor and Mesh networks.

T. G. Basavaraju has obtained his B.E degree in Computer Science and Engineering from Kuvempu University, Shimoga, Karnataka, M.E. degree in Computer Science and Engineering, University Visvesvaraya College of Engineering, Bangalore University, Bangalore, Karnataka and $\mathrm{Ph}$.D. degree from Jadavpur University, Kolkata. Currently, he is working as Professor and Head, Department of Computer Science and Engineering, Government SKSJ Technological Institute, Bangalore. He has more than 20 years of teaching and industrial experience. He has published several papers in national and international journals. He has also authored and coauthored several text books in the area of Computer Networking. His research interests are Wireless Mobile Ad hoc Networks, Wireless Sensor Networks and Wireless Mesh Networks. 